\title{
Revascularization Evaluation of Autologous Fascia Lata Graft Following Shoulder Superior Capsule Reconstruction by Enhanced Magnetic Resonance Imaging
}

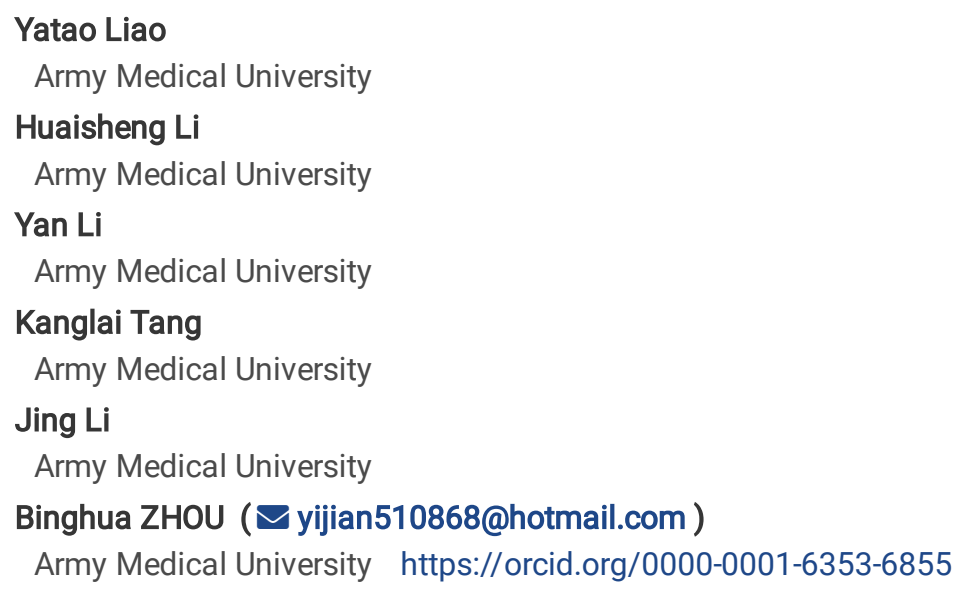

\section{Research Article}

Keywords: Revascularization, Graft, Superior capsule, Reconstruction, Magnetic resonance imaging

Posted Date: February 2nd, 2022

DOl: https://doi.org/10.21203/rs.3.rs-1271738/v1

License: (c) (1) This work is licensed under a Creative Commons Attribution 4.0 International License. Read Full License 


\section{Abstract \\ Background}

Fascia lata has been used for arthroscopically superior capsule reconstruction (ASCR) and verified to achieve a good clinical outcome. However, it is still not known how the fascia lata revascularizes after ASCR. This study was performed to evaluate the revascularization of autologous fascia lata grafts after ASCR by enhanced magnetic resonance imaging (MRI).

\section{Methods}

A prospective study of 13 patients with irreparable rotator cuff tears underwent ASCR with autologous fascia lata grafts from September 2019 to December 2020. Radiography examinations and clinical evaluations were performed preoperatively and postoperatively at 6 weeks and 3, 6, and 12 months. The signal-to-noise quotient (SNQ) value and enhancement index (EI) of autologous fascia lata grafts in the great tubercle insertion (GTI), midpoint of the graft (MG) and glenoid insertion (GI) were compared for radiography examination. Clinical evaluation included the American Shoulder and Elbow Surgeons (ASES) score, University of California Los Angeles (UCLA) shoulder score and Visual Analog Scale (VAS) score.

\section{Results}

The SNQ values in T1WI enhancement at GI and GTI were significantly higher than those at the plain MRI scan at all observation timepoints; however, the SNQ values in T1WI enhancement at MG did not show a significant difference until 3 months postoperation. EI values at GTI and GI were significantly higher than those at MG at 6 weeks and 3 months postoperation, while there was no significant difference in the El value between GTI and GI. At 12 months postoperation, the El value at GI was significantly higher than those at MG and GTI; however, there was no significant difference between GTI and MG. The El values at GTI and MG peaked at 3 months and 6 months postoperation, respectively, and then plateaued at 12 months postoperation. However, there was no significant difference in the EI value among the different timepoints at the GI. The El value did not correlate with the VAS scores at any time point or any observation location.

\section{Conclusion}

Revascularization of the fascia lata is dependent on the location of the fascia lata and plateaus at 12 months postoperation. The El value did not correlate with the VAS scores at any time point or any observation location.

\section{Introduction}

Arthroscopic superior capsule reconstruction (ASCR) using autologous fascia lata or dermal allografts has been demonstrated to be a beneficial treatment method for irreparable massive rotator cuff tears ${ }^{[1-3]}$, and ASCR has achieved good short and medium-term clinical effects $^{[4-8]}$. ASCR improves shoulder function by restoring the superior stability of the shoulder biomechanically ${ }^{[9]}$. However, restoring superior stability relies on good fascial bone healing in both the glenoid and rotator cuff footprints, as well as the continuity of the graft.

The fascia lata is rich in blood vessel tissue ${ }^{[10]}$. Previous research has shown that angiogenesis was important to tendon-to-bone healing ${ }^{[11]}$. Moreover, angiogenesis was parabolic during the fascial bone healing process in a rat massive rotator cuff model ${ }^{[12]}$. However, the exact revascularization process of autologous fascia lata grafts following shoulder ASCR is not clearly understood, and there is no clinical report on its evaluation.

Magnetic resonance imaging (MRI) plain scans are the gold standard for the diagnosis of rotator cuff tears ${ }^{[13]}$; however, plain MRI scans cannot identify blood flow in the graft. Enhanced MRI can display blood flow directly when paramagnetic contrast agents such as GdDTPA enter the observed target tissue through blood flow after injection, and the signal values of enhanced MRI changed with the intensity and position of autograft revascularization during the healing process in tendon-to-bone areas ${ }^{[14-17]}$. However, to date, there has been no radiological evaluation of revascularization of autologous fascia lata grafts after ASCR.

To clarify the characteristics of graft revascularization after ASCR, in this study, we first compared the signal intensity in both plain MRI scans and enhanced MRI to investigate whether enhanced MRI could reveal blood flow in the autogenous fascia lata. Then, we 
prospectively analyzed the blood circulation in the glenoid, humeral head insertion and the midpoint of the graft at different time points postoperation.

\section{Materials And Methods}

This study was approved by the Ethics Committee of Southwest Hospital (No: KY20202128). From September 2019 to December 2020, the senior author $(\mathrm{ZBH})$ performed all ASCR evaluations in 30 patients, and 13 patients were enrolled in this study (power=0.8; sig. lever=0.05; $d=0.8$ ). The inclusion criteria were as follows: (1) irreparable massive rotator cuff tears that required ASCR using autologous fascia lata; (2) a follow-up time longer than 12 months; and (3) plain T1WI scans and enhanced MRI performed at all follow-up time points. We defined the exclusion criteria as follows: (1) severe internal diseases, such as heart failure et al; and (2) graft rupture shown on postoperative MRI.

\section{MRI Scan}

All patients were examined by a 1.5T magnetic resonance scanner (Siemens Magneton Essensa) through a special coil for the shoulder joint. The patients were placed in the supine neutral position, with the opposite injured shoulder joint raised $30^{\circ}$. Then, the palm of the injured arm was placed facing upward, the shoulder was kept in the center of the examination bed as much as possible, and sandbags were laterally placed to prevent movement. An infusion tube was placed in the contralateral anterior elbow vein before examination and rendered the patient completely motionless during examination. Finally, the center was aligned with the coil center and humeral head. The scanning sequence included a plain scan and enhanced scan of the injured shoulder. Plain MRI scan sequences were conducted considering the following parameters: 1) PD-TSE-Dixon coronal position, 2) T1WI-TSE coronal position (scan baseline: parallel to the long axis of the scapula in the transverse position and parallel to the long axis of the humerus in the sagittal position), and 3) T2-TSE-Dixon transverse position. Enhanced MRI sequences were conducted considering the following parameters: 1) T1WI-TSE coronal position (scanning parameters were identical to those of T1WI-TSE plain scanning), 2) T1WI-TSE-FS transverse position, and 3) T1WI-TSE-FS sagittal position (scanning baseline: transverse position ran perpendicular to the long axis of the supraspinatus tendon, while coronal position ran parallel to the long axis of the humerus). Later, an enhanced MRI scan was performed, gadolinium (GdDTPA) contrast agent was injected intravenously at $0.1 \mathrm{mmol} / \mathrm{kg}$, and a T1WI-TSE coronal scan was performed 5 minutes after injection to achieve the best effect. During the whole data acquisition period, the parameters of the MRI scanning sequences (Table 1) were maintained.

\section{MRI Measurement}

A Siemens Syngo postprocessing system was used for image analysis. The SNQ values of 5 consecutive slices with good graft integrity were measured on both plain scan and enhanced MRI-T1WI-TSE coronal images. First, the signal value was calibrated by measuring the signal value of the same blank position of the plain T1WI scan and enhancement. Then, the magnetic resonance signal intensities of three parts of the graft (GTI, MG and GI) were measured (fig 1). The FOV selected sites and areas of both plain and enhanced T1WI images were the same. The average value of the 5-slice signal in each FOV region was calculated, and the signal-to-noise ratio (signal-tonoise ratio $=$ measured site signal value/background (blank position) signal value) at 3 points was calculated ${ }^{[15]}$. Finally, the enhancement index (El: enhanced SNQ value/plain scan SNQ value) of each point of the graft was calculated. Data measurement and analysis at all selected levels were carried out in exactly the same way. By comparing the changes in signal intensity and El at the same site between plain scans and enhancement at different time points, indirect information about revascularization was provided to reflect the blood supply of the graft. VAS scores were used to quantitatively score the pain at different postoperative times. ASES and UCLA scores were used to evaluate the postoperative function of the patient at 6 months and 12 months.

\section{Surgical Technique}

ASCR was performed using the technique described by Mihata ${ }^{[6,18]}$. We performed all procedures using general anesthesia with the patient in the lateral decubitus position. Normal pump pressure was set between 30 and $50 \mathrm{mmHg}$. We established a posterior portal for initial assessment of the glenohumeral joint and then established an anterior portal through the rotator interval for the treatment of intraarticular lesions, such as labral tears, and repaired the torn subscapularis tendon. We also established a lateral portal and removed any pathologic bursal tissue, performed subacromial decompression to create a flat acromial undersurface and debrided the superior glenoid and rotator cuff footprint of the greater tuberosity to expose cortical bone.

\section{Measured capsular defect size and harvested fascia lata grafts}


The size of the superior was evaluated with a measuring probe in both the anteroposterior (from the anterior edge to the posterior edge of the torn tendon) and mediolateral (from the superior edge of the glenoid to the lateral edge of the greater tuberosity) directions at $30^{\circ}$ of shoulder abduction. We made a vertical skin incision over the lateral thigh around the greater trochanter of the femur and harvested the fascia lata. The optimal graft length in the anteroposterior direction was exactly the same as the length of the defect. The graft length in the mediolateral direction was $20 \mathrm{~mm}$ longer than the distance from the superior edge of the glenoid to the lateral edge of the greater tuberosity to give a 5 -mm footprint on the superior glenoid and a $15 \mathrm{~mm}$ footprint on the great tubercle.

\section{Graft attachment}

The graft was fixed to the neck of the glenoid using two anchors (diameter, $5.5 \mathrm{~mm}$, Hearlix, Depuy Mitek, USA) at the 10-11 o'clock and 12-1 o'clock positions in the right shoulder (or the 1-2 o'clock and 11-12 o'clock positions in the left shoulder). The graft was then inserted through the anterolateral portal into the subacromial space directly. When the medial edge of the graft had reached the superior glenoid, all NO. 2 Orthorcords were tied. We attached the lateral side of the fascia lata to the rotator cuff footprint on the greater tuberosity by using the compression double-row technique (2 $5.5 \mathrm{~mm}$ Healix advance and 2 Versalok). Residual infraspinatus tissue was managed with posterior convergence in all patients after fixation of the graft.

\section{Postoperative Protocol}

We recommend the use of an abduction airbag for 6 weeks after reconstruction. After the immobilization period, passive and activeassisted exercises were initiated to promote "scaption" (scapular plane elevation). Three months after surgery, patients began to perform exercises to strengthen the rotator cuff and the scapula stabilizers. Physical therapists assisted all patients.

\section{Statistical Analysis}

Statistical analyses were performed with SPSS 22. The average signal values of five layers in each part at different time points were measured, and SNQ and El values were calculated. Normality of the data sets was assessed using the Kolmogorov-Smirnov and Shapiro-Wilk tests, and appropriate paired Student's $t$ tests or Wilcoxon signed rank tests were then conducted depending on the results of normality testing. The relationship between the VAS, ASES, or UCLA score and El was analyzed by bivariate correlation analysis.

\section{Results}

\section{Enhanced MRI could reveal the blood flow in autologous fascia lata grafts after ASCR}

To verify that enhanced MRI scans could reveal the revascularization process of the autologous fascia lata grafts, we compared all 13 patients' MRI plains and enhanced MRI scans of 3 locations at 6 weeks, 3 months, 6 months and 12 months postoperation. The SNQ values of the autologous fascia lata grafts at the greater tubercle, midpoint of the fascia lata and insertion of the glenoid from enhanced MRI examination were significantly higher than those from plain T1Wl, except for the comparison at the midpoint of the fascia lata grafts at 6 weeks postoperation (Table 2).

\section{Revascularization of different locations at the autologous fascia lata grafts}

We investigated the revascularization of different locations at the autologous fascia lata grafts at the same timepoint. At 6 weeks and 3 months postoperation, the El value of the great tubercle and glenoid insertion were significantly higher than those at the midpoint of the graft. At 6 months postoperation, there was no significant difference in the El value among the glenoid insertion, great tubercle, or midpoint of the graft. At 12 months postoperation, the El value on the articular glenoid side was significantly elevated compared with the middle of the graft and the greater tuberosity. (Table 3).

\section{Revascularization of autologous fascia lata grafts at different time points}

We compared the El of autologous fascia lata grafts at different time points after ASCR to analyze the change in graft revascularization at the same location over time. At the great tubercle, the El value first increased and peaked at 3 months, then decreased and finally plateaued at 12 months postoperation. The El showed a significant increase at 3 months postoperation compared with that at 6 weeks postoperation. Compared with 3 months postoperation, there was a significant decrease at 6 months and 12 months postoperation, while no significant difference between 12 months and 6 months postoperation was detected.

At the midpoint of the graft, the El value peaked at 6 months postoperation and plateaued at 12 months postoperation. The El value was significantly increased at 3, 6 and 12 months compared with 6 weeks postoperation. Compared with 3 months postoperation, the El at 6 
months and 12 months postoperation was significantly increased; however, there was no significant difference between those at 6 months and 12 months postoperation.

At glenoid insertion, there was no significant difference in the El value among the different timepoints. No significant difference was shown in the El value among 6 weeks and 3, 6 and 12 months postoperation (Table 4 and Fig 2).

\section{Correlation between shoulder function and revascularization of autologous fascia lata grafts}

The VAS was used to evaluate the degree of postoperative pain, the ASES and UCLA were used to evaluate postoperative functional recovery, and the correlation between the VAS, ASES or UCLA score and the El at different time points and different parts was analyzed. The results showed no significant correlation between the VAS score and El value at any time point and any observation location (Table 5). The El value at the midpoint of the graft 6 months postoperation was positively correlated with the ASES score, and the El value at the great tubercle 12 postoperation was negatively correlated with the UCLA score (Table 6).

\section{Discussion}

ASCR has been suggested to be a clinical choice for irreparable massive rotator cuff tears ${ }^{[5,19]}$. The aim of ASCR is to restore the superior stability of the shoulder biomechanically, and the most important aspect is to achieve good fascial bone healing in both the glenoid insertion and rotator cuff footprint. Autograft revascularization is a key factor for fascial bone healing and good clinical outcome. Therefore, we evaluated the revascularization of fascia lata grafts after ASCR through enhanced MRI.

Enhanced MRI can evaluate revascularization in fascia lata grafts after ASCR. As a noninvasive imaging method, MRI has been widely used in the diagnosis of different types of shoulder diseases. It can not only be used in the diagnosis of rotator cuff tears but also reflect the blood supply of rotator cuffs by comparing the changes in magnetic resonance SNQ values ${ }^{[20]}$. Sasanuma H. found that shoulder pain and limited movement were related to abnormal hemodynamics by dynamic magnetic resonance imaging ${ }^{[21,22]}$. Kim S. H found inferior tendon perfusion immediately after repair ${ }^{[14]}$; however, there was no long-term observation. In this study, we found that the T1WI enhancement signal values of the fascia lata grafts were significantly higher than those of the plain scan at different postoperative timepoints, except at the midpoint of the fascia lata 6 weeks postoperation. We reasonably assert that revascularization of the fascia lata at the glenoid insertion and great tubercle appeared at 6 weeks postoperation but not at the midpoint of the fascia lata. Enhanced MRI can be used to evaluate the revascularization of fascia lata grafts after ASCR.

The revascularization of the fascia lata changed over time. The El of the fascia lata in the great tubercle increased significantly at 6 weeks postoperation and peaked at 3 months postoperation, and there was no significant difference between 6 months and 12 months postoperation. This finding supported that angiogenesis was parabolic and progressed downward during the fascial bone healing process in a rat massive rotator cuff model ${ }^{[12]}$. Angiogenesis might be helpful for the healing of the fascia-to-bone interface in the early phase. When healing was complete, inflammation and angiogenesis receded. The El of the fascia lata at the midpoint of the fascia lata peaked at 6 months postoperation and plateaued at 12 months postoperation, which means that revascularization was late compared at the great tubercle and glenoid insertion. However, at the glenoid, there was no significant difference in the El value among the different timepoints. Therefore, it is reasonable to speculate that the revascularization of the fascia lata at the glenoid was stable in the early stage of ASCR. In summary, revascularization of the fascia lata was time-dependent, and revascularization of the fascia lata first appeared at the glenoid insertion and great tubercle and then extended to the midpoint of the graft.

The biomechanical basis of ASCR is to restore the superior stability of the shoulder joint through better fascia-to-bone healing. Benke et al. believed that in the surgical repair of rotator cuff tears, simple suturing of the broken end should not be adopted. Instead, the ischemic tissue of the broken end should be excised, and then sutured or appropriate tendon substitute materials should be used for repair, which is conducive to healing and better long-term efficacy by improving the local blood supply ${ }^{[23]}$. In this study, we found that the revascularization of the fascia lata was location-dependent. The revascularization of the autogenous fascia lata grafts was first established at the end of the tubercle and glenoid and then extended to the midpoint of the graft. Revascularization of the fascia lata grafts at the glenoid insertion reached a stable state at 6 weeks postoperation; however, at the great tubercle and midpoint of the graft, it plateaued 12 months postoperation. Ntoulia A.F. found that graft revascularization first appeared in the intra-articular part and then extended to other parts of the graft after anterior cruciate ligament reconstruction and speculated that the process of revascularization was also closely related to the surrounding microenvironment ${ }^{[15]}$. Harukazu T. Y further confirmed that vascular epithelial growth factor (VEGF) played an important role in the process of revascularization ${ }^{[24]}$. Collectively, the results of this study suggested that revascularization of the fascia lata after ASCR might be formed at the glenoid and tubercle insertion first and then extend to the middle 
part of the graft. The revascularization of the fascia lata grafts was location-dependent and obviously affected by multiple factors, such as the local microenvironment and biological factors, but further research is needed.

This study has several limitations. First, only 13 patients were included in this study. However, this study was designed to be a prospective study, and power analysis showed that 13 patients were sufficient. Second, we did not show the vessels in the fascia lata graft directly; however, revascularization was evaluated by the SNQ value and El value at different time intervals and 3 different locations of the graft, and we believe the results are still robust. Further study and new techniques are needed to reveal the angiogenesis process and blood supply in fascia lata grafts directly.

In conclusion, MRI enhancement was successfully used to evaluate revascularization of the fascia lata after ASCR. Revascularization of the graft first formed at the great tuberosity and glenoid and then extended to the midpoint of the graft. Revascularization of the fascia lata grafts at the glenoid insertion reached a stable state at 6 weeks postoperation; however, at the great tubercle and midpoint of the graft, it plateaued 12 months postoperation. Postoperative pain did not correlate with graft revascularization. Revascularization at the midpoint of the graft 6 months postoperation was positively correlated with the ASES score; however, revascularization at the great tubercle at 12 months postoperation was negatively correlated with the UCLA score.

\section{Abbreviations}

\section{SCR}

superior capsule reconstruction

ASCR

arthroscopic superior capsule reconstruction

GTI

great tubercle insertion

MG

midpoint of the graft

GI

glenoid insertion

MRI

magnetic resonance imaging

SNQ

signal-to-noise quotient

EI

enhancement index

ASES

American Shoulder and Elbow Surgeons

UCLA

University of California Los Angeles

VAS

Visual Analog Scale

VEGF

vascular epithelial growth factor.

\section{Declarations}

\section{Acknowledgments}

We thanks the Dr Teruhisa Mihata' help on the tutorial guidance of surnical technique.

\section{Authors' contributions}

Ya-tao Liao collected the data. Ya-tao Liao and Huai-sheng Li analyzed the data and prepared the figures. Ya-tao Liao, Yan Li, and Jing Li drafted the initial manuscript. Jing Li and Kang-lai Tang designed the study. Bing-hua Zhou reviewed and revised the manuscript. All authors read and approved the final manuscript. 


\section{Funding}

This paper is supported by Chongqing Yingcai Projects for Creative Leading Talents (CQYC2020303135).

\section{Availability of data and materials}

The data sets used and/or analyzed during the current study are available from the corresponding author on reasonable request.

\section{Ethics approval and consent to participate}

This study was approved by the Ethics Committee of the First Affiliated Hospital of Army Medical University, PLA. All the patients signed written informed consent.

\section{Consent for publication}

Not applicable.

\section{Competing interests}

The authors declare that they have no competing interests.

\section{References}

1. Lacheta L, Horan M, Goldenberg B, Dornan G, Higgins B, Millett P. Minimum 2-year clinical outcomes after superior capsule reconstruction compared with reverse total shoulder arthroplasty for the treatment of irreparable posterosuperior rotator cuff tears in patients younger than 70 years. Journal of shoulder elbow surgery. 2020;29(12):2514-22.

2. Lacheta L, Horan M, Schairer W, Goldenberg B, Dornan G, Pogorzelski J, et al. Clinical and Imaging Outcomes After Arthroscopic Superior Capsule Reconstruction With Human Dermal Allograft for Irreparable Posterosuperior Rotator Cuff Tears: A Minimum 2-Year Follow-Up. Arthroscopy. 2020;36(4):1011-9.

3. Oh J, Park M, Rhee S. Treatment Strategy for Irreparable Rotator Cuff Tears. Clinics in orthopedic surgery. 2018;10(2):119-34.

4. Denard P, Brady P, Adams C, Tokish J. Burkhart. Preliminary Results of Arthroscopic Superior Capsule Reconstruction with Dermal Allograft. Arthroscopy. 2018;34(1):93-9.

5. Li H, Yang M, Li Y, Zhou B, Tang K. Research progress of indication and treatment of graft in shoulder superior capsular reconstruction for rotator cuff tear. Chinese journal of reparative reconstructive surgery. 2021;35(2):252-7.

6. Mihata T, Bui C, Akeda M, Cavagnaro M, Kuenzler M, Peterson A, et al. A biomechanical cadaveric study comparing superior capsule reconstruction using fascia lata allograft with human dermal allograft for irreparable rotator cuff tear. Journal of shoulder elbow surgery. 2017;26(12):2158-66.

7. Rosales-Varo A, Zafra M, García-Espona M, Flores-Ruiz M, Roda O. Superior capsular reconstruction of irreparable rotator cuff tear using autologous hamstring graft. Revista espanola de cirugia ortopedica y traumatologia. 2019;63(1):1-6.

8. Woodmass J, Wagner E, Borque K, Chang M, Welp K, Warner J. Superior capsule reconstruction using dermal allograft: early outcomes and survival. Journal of shoulder elbow surgery. 2019;28:100-S9.

9. Mihata T, Lee T, Watanabe C, Fukunishi K, Ohue M, Tsujimura T, et al. Clinical results of arthroscopic superior capsule reconstruction for irreparable rotator cuff tears. Arthroscopy. 2013;29(3):459-70.

10. Janik S, Hirtler L, Traxler H, Weninger W, Seemann R, Erovic B. The vascularized fascia lata free flap: an anatomical study and clinical considerations. Eur Arch Otorhinolaryngol. 2020;277(6):1733-9.

11. Li H, Ma L, Li Y, Tao X, Liao Y, Yang A, et al. The short-term effectiveness of superior capsular reconstruction using autologous fascia lata graft for irreparable massive rotator cuff tears. Chinese journal of reparative reconstructive surgery. 2021;35(11):1427-33.

12. Li HZM, Huang P, Liu J, Tang H, Zhang C, Wang Y, Shi YX, Liao Y, Jin B, Tang K, Zhou B. Histologic and biomechanical evaluation of the thoracolumbar fascia graft for massive rotator cuff tears in a rat model. Journal of Shoulder and Elbow Surgery. 2021.

13. Zhou B, Zhou Y, Tang K. An overview of structure, mechanical properties, and treatment for age-related tendinopathy. The journal of nutrition health aging. 2014;18(4):441-8.

14. Kim S, Cho W, Joung H, Choi Y, Jung M. Perfusion of the Rotator Cuff Tendon According to the Repair Configuration Using an Indocyanine Green Fluorescence Arthroscope: A Preliminary Report. Am J Sports Med. 2017;45(3):659-65. 
15. Ntoulia A, Papadopoulou F, Ristanis S, Argyropoulou M, Georgoulis A. Revascularization process of the bone--patellar tendon--bone autograft evaluated by contrast-enhanced magnetic resonance imaging 6 and 12 months after anterior cruciate ligament reconstruction. Am J Sports Med. 2011;39(7):1478-86.

16. Ntoulia A, Papadopoulou F, Zampeli F, Ristanis S, Argyropoulou M, Georgoulis A. Evaluation with contrast-enhanced magnetic resonance imaging of the anterior cruciate ligament graft during its healing process: a two-year prospective study. Skeletal radiology. 2013;42(4):541-52.

17. Urita A, Funakoshi T, Horie T, Nishida M, Iwasaki N. Difference in vascular patterns between transosseous-equivalent and transosseous rotator cuff repair. Journal of shoulder elbow surgery. 2017;26(1):149-56.

18. Okamura K, Abe M, Yamada Y, Makihara T, Yoshimizu T, Sakaki Y, et al. Arthroscopic superior capsule reconstruction with Teflon felt synthetic graft for irreparable massive rotator cuff tears: clinical and radiographic results at minimum 2-year follow-up. Journal of shoulder elbow surgery. 2021;30(3):625-34.

19. Liao Y, Zhou B, Mihata T. Superior capsule reconstruction: an overview of anatomy, biomechanics, indications, and graft treatment. Chinese medical journal. 2021.

20. Liu S, Xie Y, Chen Q, Sun Y, Ding Z, Zhang Y, et al. Tendon Healing Progression Evaluated With Magnetic Resonance Imaging Signal Intensity and Its Correlation With Clinical Outcomes Within 1 Year After Rotator Cuff Repair With the Suture-Bridge Technique. Am J Sports Med. 2020;48(3):697-705.

21. Sasanuma H, Sugimoto H, Fujita A, Kanaya Y, lijima $Y$, Saito T, et al. Characteristics of dynamic magnetic resonance imaging of idiopathic severe frozen shoulder. Journal of shoulder elbow surgery. 2017;26(2):e52-e7.

22. Sasanuma H, Sugimoto H, lijima Y, Kanaya Y, Saito T, Takeshita K. Blood flow evaluation by dynamic magnetic resonance imaging of symptomatic rotator cuff tears and frozen shoulders. Journal of shoulder elbow surgery. 2018;27(12):e372-e9.

23. Wong C, Ng E, Fung P, Mok K, Yung P, Chan K. Comparison of treatment effects on lateral epicondylitis between acupuncture and extracorporeal shockwave therapy. Asia-Pacific journal of sports medicine, arthroscopy, rehabilitation.technology. 2017;7:21-6.

24. Tohyama H, Yoshikawa T, Ju Y, Yasuda K. Revascularization in the tendon graft following anterior cruciate ligament reconstruction of the knee: its mechanisms and regulation. Chang Gung Med J. 2009;32(2):133-9.

\section{Tables}

Table 1

Parameters of magnetic resonance imaging sequences

\begin{tabular}{|c|c|c|c|c|c|c|}
\hline \multirow{2}{*}{$\begin{array}{l}\text { Parameter } \\
\text { sequences }\end{array}$} & \multicolumn{3}{|c|}{ MRI plain scanning } & \multicolumn{3}{|c|}{ Enhanced MRI } \\
\hline & $\begin{array}{l}\text { PD-TSE-Dixon } \\
\text { coronal position }\end{array}$ & $\begin{array}{l}\text { T1W1-TSE } \\
\text { coronal } \\
\text { position }\end{array}$ & $\begin{array}{l}\text { T2-TSE-Dixon } \\
\text { transverse } \\
\text { position }\end{array}$ & $\begin{array}{l}\text { T1W1-TSE } \\
\text { coronal } \\
\text { position }\end{array}$ & $\begin{array}{l}\text { T1W1-TSE-FS } \\
\text { transverse } \\
\text { position }\end{array}$ & $\begin{array}{l}\text { T1W1-TSE-FS } \\
\text { sagittal position }\end{array}$ \\
\hline $\mathrm{TR}(\mathrm{ms})$ & 3720 & 600 & 3000 & 600 & 616 & 724 \\
\hline TE (ms ) & 38 & 12 & 38 & 12 & 11 & 14 \\
\hline $\begin{array}{l}\text { Layer } \\
\text { Thickness ( } \\
\mathrm{mm} \text { ) }\end{array}$ & 3 & 3 & 3 & 3 & 3 & 3 \\
\hline $\begin{array}{l}\text { Layer } \\
\text { Spacing ( } \\
\mathrm{mm})\end{array}$ & 10 & 10 & 30 & 10 & 30 & 10 \\
\hline FOV $\left(\mathrm{mm}^{2}\right)$ & $180 \times 180$ & $180 \times 180$ & $180 \times 180$ & $180 \times 180$ & $180 \times 180$ & $192 \times 192$ \\
\hline $\begin{array}{l}\text { Matrix ( } \\
\left.\mathrm{mm}^{2}\right)\end{array}$ & $256 \times 204$ & $256 \times 204$ & $256 \times 204$ & $256 \times 204$ & $256 \times 204$ & $256 \times 180$ \\
\hline $\begin{array}{l}\text { Collection } \\
\text { Numbers }\end{array}$ & 2 & 2 & 1 & 2 & 1 & 1 \\
\hline $\begin{array}{l}\text { Collection } \\
\text { Time } \\
\text { (min:s) }\end{array}$ & $3: 21$ & $2: 53$ & $1: 53$ & $3: 21$ & $1: 30$ & $2: 15$ \\
\hline
\end{tabular}


Table 2

SNQ values in different position between the plain scan and the enhanced phase of the T1WI at different time points postoperative.

\begin{tabular}{|c|c|c|c|c|c|c|c|c|c|}
\hline & \multicolumn{3}{|c|}{ Greater Tubercle } & \multicolumn{3}{|c|}{ Midpoint of Graft } & \multicolumn{3}{|c|}{ Glenoid Insertion } \\
\hline & $\begin{array}{l}\text { T1WI Plain } \\
\text { Scan }\end{array}$ & $\begin{array}{l}\text { T1WI } \\
\text { enhancement }\end{array}$ & $\begin{array}{l}P \\
\text { value }\end{array}$ & $\begin{array}{l}\text { T1WI Plain } \\
\text { Scan }\end{array}$ & $\begin{array}{l}\text { T1WI } \\
\text { enhancement }\end{array}$ & $\begin{array}{l}\mathrm{P} \\
\text { value }\end{array}$ & $\begin{array}{l}\text { T1WI Plain } \\
\text { Scan }\end{array}$ & $\begin{array}{l}\text { T1WI } \\
\text { enhancement }\end{array}$ & $\begin{array}{l}\mathrm{P} \\
\text { value }\end{array}$ \\
\hline $\begin{array}{l}6 \\
\text { weeks }\end{array}$ & $28.98 \pm 8.81$ & $49.52 \pm 27.44$ & 0.000 & $29.23 \pm 8.95$ & $31.32 \pm 10.69$ & 0.329 & $29.26 \pm 6.83$ & $53.34 \pm 24.37$ & 0.000 \\
\hline $\begin{array}{l}3 \\
\text { months }\end{array}$ & $26.09 \pm 4.92$ & $53.81 \pm 16.43$ & 0.000 & $25.53 \pm 6.35$ & $38.01 \pm 14.84$ & 0.000 & $27.31 \pm 7.11$ & $54.57 \pm 20.05$ & 0.000 \\
\hline $\begin{array}{l}6 \\
\text { months }\end{array}$ & $26.94 \pm 6.91$ & $48.34 \pm 12.35$ & 0.000 & $25.39 \pm 8.19$ & $44.62 \pm 19.15$ & 0.000 & $26.80 \pm 6.68$ & $52.40 \pm 17.37$ & 0.000 \\
\hline $\begin{array}{l}12 \\
\text { months }\end{array}$ & $26.44 \pm 13.42$ & $38.54 \pm 13.88$ & 0.000 & $25.95 \pm 13.34$ & $39.61 \pm 21.58$ & 0.000 & $25.36 \pm 16.57$ & $45.80 \pm 24.07$ & 0.000 \\
\hline
\end{tabular}

Table 3 The comparation of enhancement index in different position after superior capsule reconstruction

\begin{tabular}{|llll|}
\hline & GT & MG & GI \\
\hline 6 weeks & $1.51 \pm 0.46^{\#}$ & $1.07 \pm 0.19$ & $1.67 \pm 0.51^{\#}$ \\
\hline 3 months & $2.04 \pm 0.37^{\#}$ & $1.20 \pm 0.23$ & $1.95 \pm 0.39^{\#}$ \\
\hline 6 months & $1.84 \pm 0.26$ & $1.78 \pm 0.40$ & $1.94 \pm 0.37$ \\
\hline 12 months & $1.57 \pm 0.30$ & $1.56 \pm 0.36$ & $1.87 \pm 0.31^{\text {*\# }}$ \\
\hline
\end{tabular}

GT: Greater Tubercle; MG: Midpoint of Graft; GI: Glenoid Insertion.

* Compared with GT, P $<0.05$; ${ }^{\#}$ Compared with MG, P $<0.05$

Table 4 The comparation of enhancement index value at different time points after superior capsule reconstruction

\begin{tabular}{|lllll|}
\hline & 6 weeks & 3 months & 6 months & 12 months \\
\hline GT & $1.51 \pm 0.46$ & $2.04 \pm 0.37^{\mathrm{a}}$ & $1.84 \pm 0.26^{\mathrm{a} \beta}$ & $1.55 \pm 0.31^{\beta}$ \\
MG & $1.07 \pm 0.19$ & $1.20 \pm 0.23^{\mathrm{a}}$ & $1.78 \pm 0.40^{\mathrm{a} \beta}$ & $1.56 \pm 0.36^{\mathrm{a} \beta}$ \\
\hline GI & $1.67 \pm 0.51$ & $1.95 \pm 0.39$ & $1.94 \pm 0.37$ & $1.87 \pm 0.31$ \\
\hline
\end{tabular}

GT: Greater Tubercle; MG: Midpoint of Graft; GI: Glenoid Insertion.

${ }^{a}$ Compared with 6 weeks postoperative, $\mathrm{P}<0.05 ;{ }^{\beta}$ Compared with 3 months postoperative, $\mathrm{P}<0.05 \rrbracket^{\vee}$ Compared with 6 months postoperative, $\mathrm{P}<0.05$ 
Table 5

The correlation between VAS and El value at different time points and different parts

\begin{tabular}{|c|c|c|c|c|c|c|c|c|}
\hline & & \multirow[t]{3}{*}{ VAS score } & \multicolumn{6}{|l|}{ El value } \\
\hline & & & \multicolumn{2}{|l|}{$\mathrm{GT}$} & \multicolumn{2}{|l|}{ MG } & \multicolumn{2}{|l|}{ G } \\
\hline & & & Spearman & $P$ & Spearman & $\mathrm{P}$ & Spearman & $\mathrm{P}$ \\
\hline \multirow[t]{4}{*}{ VAS } & 6 weeks & $3.80 \pm 1.30$ & 0.026 & 0.966 & -0.154 & 0.805 & 0.051 & 0.935 \\
\hline & 3 months & $2.75 \pm 0.50$ & -0.775 & 0.225 & -0.775 & 0.225 & -0.775 & 0.225 \\
\hline & 6 months & $1.60 \pm 0.55$ & 0.289 & 0.638 & -0.577 & 0.308 & 0.000 & 1.000 \\
\hline & 12 months & $1.14 \pm 0.38$ & 0.412 & 0.358 & -0.612 & 0.144 & 0.000 & 1.000 \\
\hline \multicolumn{9}{|c|}{ GT: Greater Tubercle; MG: Midpoint of Graft; GI: Glenoid Insertion. } \\
\hline
\end{tabular}

Table 6

The correlation between ASES, UCLA and enhancement index at 6 months and 12months after operation

\begin{tabular}{|c|c|c|c|c|c|c|c|}
\hline & & \multicolumn{6}{|l|}{ El value } \\
\hline & & \multicolumn{2}{|l|}{ GT } & \multicolumn{2}{|l|}{ MG } & \multicolumn{2}{|l|}{ G } \\
\hline & & Spearman & $P$ & Spearman & $P$ & Spearman & $P$ \\
\hline ASES & 6 months & -0.600 & 0.285 & 0.900 & 0.037 & 0.300 & 0.624 \\
\hline \multirow[t]{3}{*}{ UCLA } & 12 months & -0.345 & 0.448 & 0.595 & 0.159 & -0.342 & 0.452 \\
\hline & 6 months & -0.205 & 0.741 & 0.667 & 0.219 & 0.051 & 0.935 \\
\hline & 12 months & -0.780 & 0.039 & 0.218 & 0.638 & -0.400 & 0.374 \\
\hline \multicolumn{8}{|c|}{ GT: Greater Tubercle; MG: Midpoint of Graft; GI: Glenoid Insertion. } \\
\hline \multicolumn{8}{|c|}{$P<0.05$ was statistically significant } \\
\hline
\end{tabular}

\section{Figures}




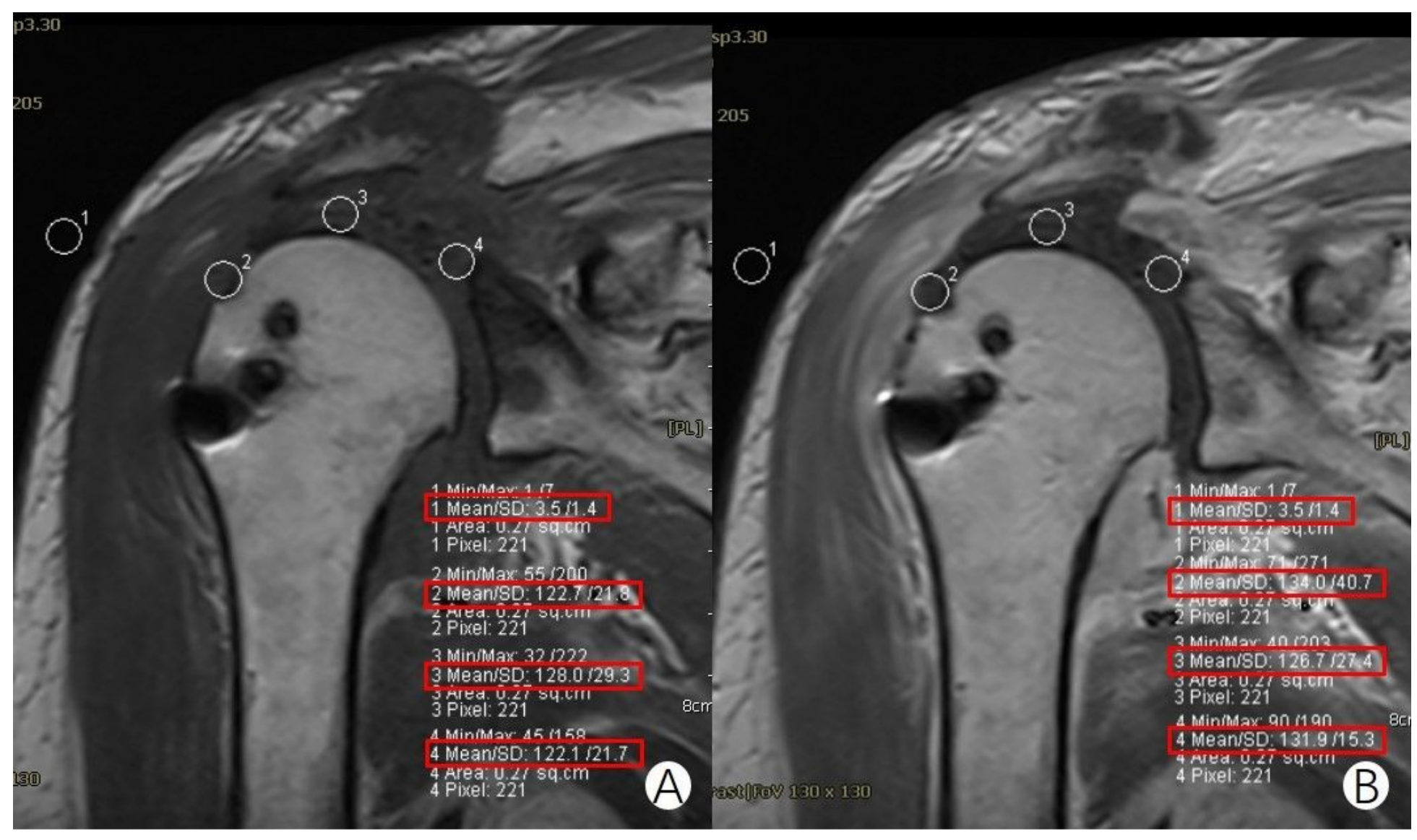

\section{Figure 1}

SNQ values were measured in T1WI plain magnetic resonance imaging (MRI) and enhanced MRI scans. A: T1WI-TSE-coronal plain scan; B: Enhanced T1WI-TSE-coronal position. Red rectangle 1 in both A and B shows that the signal values in the blank areas of the plain scan and enhancement phases were the same. Red rectangles 2,3 , and 4 show the measurement of signal intensity at three different positions (GTI, MG and $\mathrm{GI})$. 
Greater Tubercle

-Midpoint of Graft

Glenoid Insertion

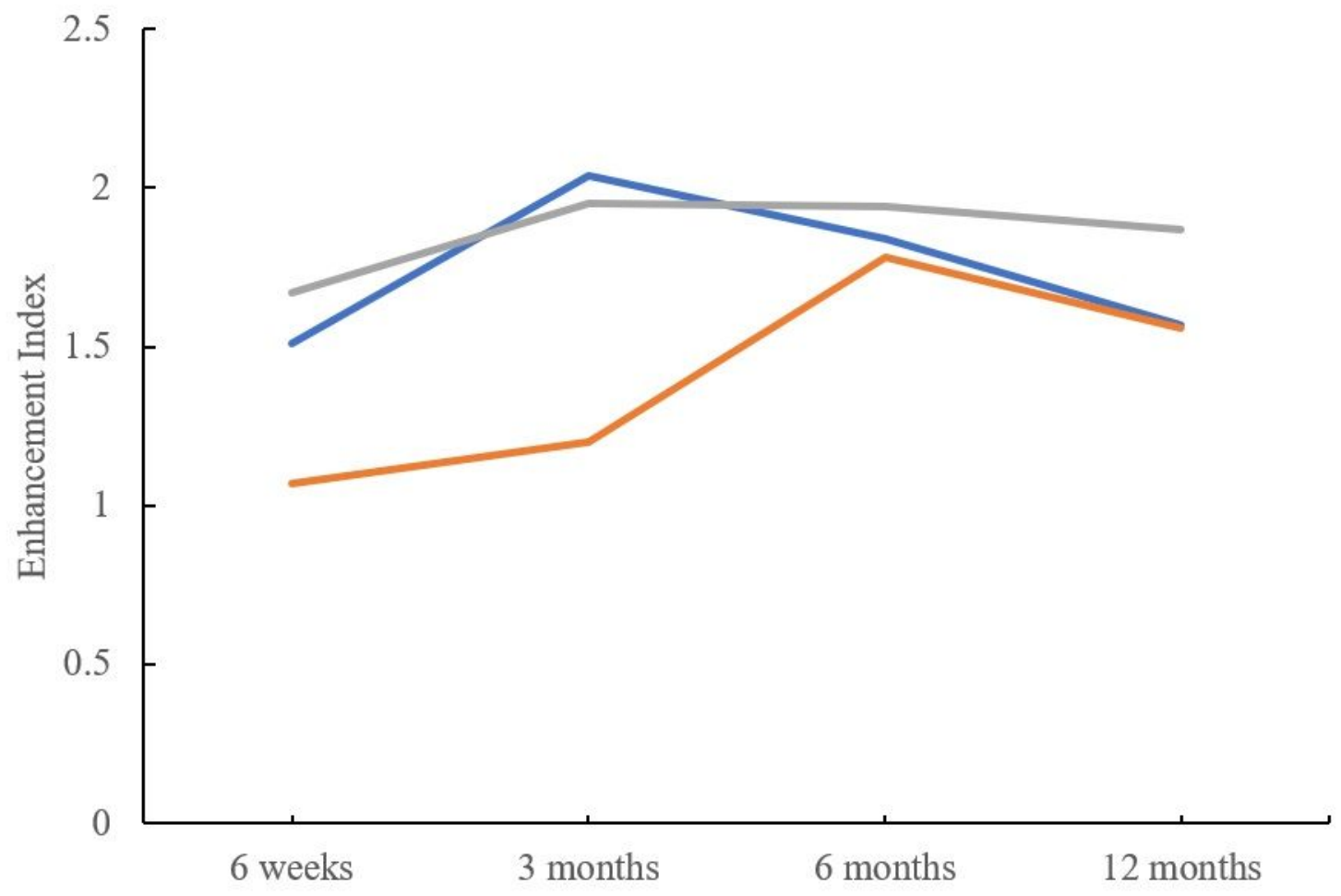

\section{Figure 2}

Comparison of the enhancement index of the graft at different time points at the great tubercle, the midpoint of the graft and the glenoid insertion. 